Rev. Elev. Méd. vét. Pays trop., 1979, 32 (4) : 347-352.

\title{
Localisation des parasites dans l'estomac du cheval de la région de Settat (Maroc)
}

\author{
par H. OUHELli $\left({ }^{*}\right)$, J. CABARET $(*)$, V. S. PANDEY $\left({ }^{* *}\right)$ et A. ELKHALFANE $\left(^{*}\right)$
}

\begin{abstract}
RESSUMÉ
L'examen de quatre-vingt-seize estomacs de chevaux originaires de la région de Settat (Maroc) a été réalisé d'octobre 1977 à septembre 1978. Chaque espèce parasitaire présente une niche particulière dans l'estomac. Ainsi Gasterophilus intestinalis est localisé essentiellement sur la muqueuse œesophagienne de l'estomac et Gasterophilus nasalis sur la muqueuse pylorique ; Trichostrongylus axei est particulièrement rencontré sur la muqueuse fundique et $\mathrm{Habro-}$ nema spp. sur la muqueuse pylorique. En règle générale, les densités élevées d'un genre parasite provoquent une extension de sa niche écologique ; au contraire les densités élevées des autres genres concurrents imposent un retrait vers la niche habituelle.
\end{abstract}

\section{INTRODUCTION}

La connaissance des localisations des parasites présente plusieurs intérêts. Elle permet de rattacher certaines lésions peu spécifiques à un type de parasite et de mieux comprendre la pathogénie des diverses espèces en cause.

Une niche écologique peut se définır, selon LEVIEUX (10), de plusieurs façons. On distingue :

- la niche fonctionnelle qui est le statut d'un organisme dans son écosystème. Elle fait intervenir divers facteurs tels la taille du parasite, son mode d'alimentation ;

- la niche topographique qui est l'expression d'un concept purement spatial ;

- la niche fondamentale qui est définie comme hypervolume à $\mathrm{n}$ dimensions. Chaque facteur enregistré constitue une dimension; ce

(*) Département de Parasitologie. Institut Agronomique et Vétérinaire Hassan 1I, B. P. 704, Rabat, Agdal (Maroc).

(**) Expert F. A. O. pourra être le pH, la densité intra- et interspécifique.

Plusieurs travaux ont contribué à cerner la niche écologique des parasites du tractus digestif des ruminants (18), des animaux de laboratoire $(1,2,11,18)$. Une étude particulièrement détaillée a concerné certaines espèces qui colonisent le gros intestin des équins (15).

Nous tenterons dans cette étude de délimiter les niches topographiques des diverses espèces parasites de l'estomac du cheval sur les trois zones histologiquement et physiologiquement variables. Certains paramètres de l'hypervolume seront appréciés à savoir le $\mathrm{pH}$ des différentes muqueuses et les densités parasitaires.

\section{MATÉRIEL ET MÉTHODES}

\section{Nature des observations}

- Les animaux: les estomacs de quatrevingt-seize chevaux de race commune, de sexe et âge différents et tous originaires de la plaine de Chaouia (Settat-Maroc), sont prélevés après abattage. 
Les prélèvements se sont étalés sur une période d'une année, allant du mois d'octobre 1977 au mois de septembre 1978 ; la fréquence des prélèvements est de six à neuf estomacs par mois.

Chaque estomac est subdivisé en trois parties, en fonction de la nature de la muqueuse :

- muqueuse proventriculaire ou csophagienne,

- muqueuse fundique,

- muqueuse antrale ou pylorique (y compris le bulbe duodénal qui constitue le prolongement du nid de Gasterophilus nasalis).

Sur soixante-six chevaux, le $\mathrm{pH}$ de chaque partie est pris immédiatement après abattage à l'aide d'un papier pH. Toutes les larves de Gasterophilus spp. ont été récoltées et identifiées selon la clé de ZUMPT (20). Trichostrongylus axei et Habronema spp. ont été récoltés sur une partie aliquote $(1 / 5$ au 1/20) du contenu stomacal en fonction de la densité parasitaire dans l'échantillon; leur identification est basée sur la clé de LICHTENFELS (12).

\section{- Méthodes d'analyse des données}

Les comparaisons de pourcentage, les méthodes de régression sont classiques (17).

Pour mesurer l'agrégation des distributions, deux indices sont utilisés: le coefficient de NEYMAN k et l'indice de MORISITA (19). Le premier coefficient est compris entre 0 et 1 et le second est supérieur à 2 pour les distributions surdispersées. Une technique plus fine, celle d'IWAO et KUNO (8), a été utilisée pour déterminer l'agrégation (indice $\beta$ ), mesurer le degré d'attraction entre les individus ( $\alpha$ ) et établir la densité environnementale. Divers modèles ont été utilisés pour apprécier la disposition des espèces et leur hiérarchie dans les niches écologiques $(6,7)$. L'inffuence du $\mathrm{pH}$ sur la disposition nidale des parasites a été estimée par la notion de sensibilité (9).

\section{LES RÉSULTATS}

Les résultats consignant la localisation des parasites sont présentés dans le tableau $\mathrm{I}$.

TABLEAU $\mathrm{N}^{\circ} \mathrm{I}$-Voyennes et êcarts-type des espèces parasites au niveau de chaque partie de $1^{\dagger}$ estorac.

\begin{tabular}{|c|c|c|c|c|c|c|}
\hline \multirow[b]{2}{*}{ Espèce parasite } & \multicolumn{2}{|c|}{$\begin{array}{c}\text { Muqueuse } \\
\text { oesophagienne }\end{array}$} & \multicolumn{2}{|c|}{$\begin{array}{l}\text { Muqueuse } \\
\text { pylorique }\end{array}$} & \multicolumn{2}{|c|}{$\begin{array}{l}\text { Muqueuse } \\
\text { fundique }\end{array}$} \\
\hline & $\begin{array}{l}\text { Moyenne } \\
\overline{\mathrm{X}}\end{array}$ & $\begin{array}{c}\text { Ecart-type } \\
s\end{array}$ & $\bar{x}$ & $s$ & $\bar{X}$ & $s$ \\
\hline $\begin{array}{l}G . \text { intestinatis } \\
\left(\mathrm{L}_{3}\right)\end{array}$ & 151,1 & 154,5 & 0,8 & 2,2 & 5,4 & 11,0 \\
\hline 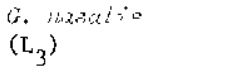 & 0,04 & 0,8 & 120,1 & 118,6 & 0,03 & 0,6 \\
\hline $\begin{array}{l}\left(\mathrm{L}_{2}\right) \\
\mathrm{inhes}\end{array}$ & 21,4 & 43,0 & - & - & - & - \\
\hline $\begin{array}{l}\text { G. thaglis } \\
\left(\mathrm{L}_{2}\right)\end{array}$ & - & - & 33,1 & 60,1 & - & - \\
\hline $\begin{array}{l}\text { 1. muscue } \\
\text { (mâles) }\end{array}$ & 2,96 & 4,4 & 64,39 & 132,6 & 42,82 & 74,2 \\
\hline $\begin{array}{l}\text { I. mi_ }=20 s \text { tom } \\
\text { (mâles) }\end{array}$ & 0,85 & 1,6 & 9,27 & 26,6 & 9,73 & 22,2 \\
\hline $\begin{array}{l}\text { Habronema spp } \\
\text { (immatures) }\end{array}$ & 24,8 & 39,5 & 408,7 & 595,5 & 206,2 & 287,6 \\
\hline $\begin{array}{l}\text { Aubronema app } \\
\text { (femelles) }\end{array}$ & 8,7 & 10,4 & 204,3 & $375, \dot{4}$ & 139,2 & 206,2 \\
\hline $\begin{array}{l}\text { T. axei } \\
\text { (mâles) }\end{array}$ & 5,64 & 14,8 & 35,57 & 133,0 & 90,37 & 258,5 \\
\hline $\begin{array}{l}\text { N. aree } \\
\text { (femelles) }\end{array}$ & 15,62 & 28,6 & 78,29 & 250,8 & 203,03 & 605,6 \\
\hline
\end{tabular}




\section{DISCUSSION}

\section{Distribution nidale des différentes espèces}

- L'examen du tableau I permet de dégager les niches topographiques suivantes:

- muqueuse asophagienne: Gasterophilus intestinalis (larves de $2^{\mathrm{e}}$ et $3^{\mathrm{e}}$ âge) en analogie avec d'autres constatations (4);

- muqueuse fundique : Trichostrongylus axei, Habronema spp. ;

- muqueuse pylorique : G. nasalis (larves de $2^{\circ}$ et $3^{\circ}$ âge), T. axei, Habronema spp.

La comparaison des densités environnementales $(\dot{m})$ permet de préciser ces localisations. La densité environnementale est un indice qui combine la moyenne et la variance (8). L'hypothèse sous-jacente est que l'agglutination est d'autant plus importante que le parasite est situé dans sa niche écologique. Pour tous les Habronema, on observe une dominance nette dans la zone pylorique. La spécificité nidale des habronèmes femelles et des immatures est plus faible que celle des mâles. La prédominance de la zone fundique pour $T$. axei est confirmée (16). La spécificité nidale chez cette espèce est plus importante chez les vers femelles, à l'opposé de ce qui est constaté chez les habronèmes. La muqueuse fundique constitue la niche topographique de $T$. axei et la muqueuse pylorique celle de Habronema spp. et $G$. nasalis.

- La dispersion des différents parasites dans les trois zones est à peu près identique pour l'ensemble des équins examinés: l'indice de MORISITA moyen (19) varie seulement de 95 à 100 suivant les muqueuses. Il est donc possible de considérer chaque zone comme un échantillon et d'appliquer la technique de IWAO et KUNO (8) sur les valeurs moyennes des trois zones pour chaque parasite.

Les valeurs des paramètres $x$ et $\beta$ de la régression de $\dot{m}$ sur $m$ sont les suivantes :

\begin{tabular}{lrr} 
& \multicolumn{1}{c}{$\alpha$} & $\beta$ \\
G. intestinalis & 10,1 & 2,0 \\
G. nasalis & 12,9 & 1,8 \\
H. muscae (mâles) & $-18,4$ & 5,2 \\
H. microstoma (mâles) & $-2,6$ & 7,5 \\
Habronema (femelles) & $-51,2$ & 4,3 \\
Habronema (immatures) & $-4,6$ & 3,1 \\
T. axei (mâles) & 82,3 & 8,8 \\
T. axei (femelles) & $-21,5$ & 10,2
\end{tabular}

Les différentes valeurs prises par le paramètre $\beta$ indiquent que la distribution suivie par l'ensemble de ces parasites est binomiale négative. Les parasites de l'estomac du cheval, dans les trois zones, suivent une loi agrégative. Cette agrégation peut être due à des facteurs liés aux vers eux-mêmes (attirance) (1) ou au milieu (l'estomac) $(3,2)$.

L'existence d'une distribution agrégative indique que l'attirance entre les parasites du même genre devrait être la règle. Ce n'est pourtant pas le cas pour l'ensemble des Habronema spp. et les femelles de $T$. axei où il semble exister une répulsion entre les individus, le coefficient $\alpha$ étant négatif. Pour ces deux groupes, la genèse d'une distribution binomiale négative doit donc s'expliquer par des facteurs autres que l'interaction entre les vers. Les arguments d'ordre purement statistique exposés par SOUTHWOOD (19) ne correspondent pas à ce qui est connu sur les distributions de parasites (5). Il semble donc plus vraisemblable d'envisager l'influence d'un facteur agglutinant extérieur aux vers. Ce facteur externe pourrait être le mucus particulièrement abondant en région pylorique (13). L'examen microscopique montre que des "logettes» sont constituées dans le mucus qui emprisonne des débris alimentaires et que les parasites sont agglutinés dans ces «logettes". Ce facteur peut être un des éléments à l'origine de l'agglutination de Habronema spp. et de $T$. axei femelles.

- Il existe une hiérarchie des espèces dans l'occupation des niches qui peut être mise en évidence lorsque l'on considère les moyennes annuelles concernant les diverses espèces dans une même zone.

En utilisant le modèle log-linéaire de MOTOMURA (6) on obtient :

pour la muqueuse œsophagienne : $\log q_{i}=2,493-0,347 x_{i} \quad(r=0,93)$

pour la muqueuse fundique :

$$
\log \mathrm{q}_{\mathrm{i}}=2,383-0,459 \mathrm{x}_{\mathrm{i}} \quad(\mathrm{r}=0,87)
$$

pour la muqueuse pylorique :

$$
\log \mathrm{q}_{\mathrm{i}}=2,988-0,272 \mathrm{x}_{\mathrm{i}} \quad(\mathrm{r}=0,9.3)
$$

avec $q_{i}$ nombre de parasites de l'espèce $i$, et $x_{i}$ rang de l'espèce $i$.

L'ajustement des mêmes données au modèle log-normal de PRESTON ou celui de Mac ARTHUR est d'une qualité identique. Chaque modèle comporte cependant des implications différentes, à savoir : 
log-linéaire : les parasites présentent un mode de vie peu différent,

log-normal : les parasites des diverses espèces dépendent d'un même ensemble de facteurs biotiques ou abiotiques,

Mac ARTHUR : les niches écologiques se touchent mais ne se recouvrent pas.

Il apparaît donc que le mode de vie de ces parasites est peu différent, qu'ils dépendent d'un même ensemble de facteurs biotiques ou abiotiques et que les niches écologiques sont assez bien individualisées.

L'ensemble des parasites de chaque zone de l'estomac du cheval constitue une nomocénose, c'est-à-dire un ensemble hiérarchisé de fonctions selon la définition de DAGET, LECORDIER et LEVÊQUE (7). Les espèces constituantes peuvent changer de rang et à la limite disparaître momentanément. La hiéra1chie est constante dans les espèces qui la constituent; seul leur ordre change.

La notion de nomocénose pourrait être utilisée au cours d'enquêtes épidémiologiques. Une nomocénose est caractéristique d'une faune parasitaire pour un organe donné. Les variations saisonnières et individuelles ne modifient pas la hiérarchie existant entre les espèces; l'utilisation du modèle log-linéaire peut permettre la comparaison de peuplements parasitaires d'un organe, dans différentes régions, bien que les prélèvements soient effectués à des périodes différentes. Le nombre de prélèvements devrait au moins atteindre la dizaine.

\section{Les yariations dans la niche écologique}

\section{- En fonction de la densité de l'espèce}

- G. intestinalis: Lorsque cette espèce existe en grand nombre au $3^{\mathrm{e}}$ stade larvaire sur la muqueuse œsophagienne, elle migre en partie vers la muqueuse fundique. Ainsi lorsque le nombre de gastérophiles sur la muqueuse œsophagienne est supérieur à la moyenne, 78,9 p. 100 des estomacs ont des larves $L_{3}$ de $G$. intestinalis sur la muqueuse fundique, à l'inverse 39,65 p. 100 en présentent lorsque la présence des larves est faible sur la muqueuse œsophagienne, la différence étant significative ( $\mathrm{p}=0,001$ ).

- G. nasalis (Larves $\mathrm{L}_{3}$ ) : Les variations de sa niche topographique ne semblent pas dépendre de sa propre densité.
- Habronema spp. (mâles, femelles et immatures) : L'analyse statistique montre une indépendance de l'étendue de sa niche écologique, vis-à-vis de ses propres variations de densité.

- Trichostrongylus axei : Lorsque le nombre de vers est faible (inférieur à la moyenne dans les trois muqueuses) les pourcentages d'infestation sont de $6,4,10,8$ et 88,8 p. 100 respectivement pour les régions œsophagienne, pylorique et fundique; si le nombre est élevé, la distribution varie comme suit: $2,6,42,3$ et 55,1 p. 100 respectivement pour les muqueuses cesophagienne, pylorique et fundique. Les différences existant entre ces 2 séries de pourcentages sont significatives $(\mathrm{p}=0,05)$.

Il est possible de conclure que si les vers mâles de l'espèce $T$. axei sont présents en grand nombre dans la muqueuse fundique, ils tendent à coloniser la muqueuse pylorique. Les vers femelles de la même espèce ne montrent pas de variation nette de leur niche écologique en fonction de leur densité.

\section{- En fonction des interactions entre espèces}

- G. intestinalis : la densité de T. axei n'influe pas sur la localisation de celte espèce ; au contraire l'augmentation de densité de Habronema spp. (formes immatures) exerce une influence nette. Il se présente deux possibilités :

1) Si G. intestinalis est en grand nombre, il évolue vers la muqueuse fundique malgré la présence de Habronema spp.;

2) $\mathrm{Si} G$. intestinalis est en faible nombre (entre 10 et 100 larves), on assiste à un retrait de la partie fundique et une concentration sur la muqueuse cesophagienne.

La densité de $G$. intestinalis n'influe pas sur la localisation de ces deux nématodes.

- Habronema spp. et Trichostrongylus axei :

Lorsque l'infestation en Habronema spp. est importante, $T$. axei augmente dans la muqueuse fundique et diminue dans la pylorique. Habronema décolonise en partie la muqueuse fundique pour se porter sur la pylorique lorsque $T$. axe est présent en grand nombre.

Il s'avère donc que plus une espèce est en grand nombre plus elle tend à occuper des niches écologiques qui ne lui sont pas particulièrement favorables et toute concurrence la ramène vers sa niche originale. 


\section{Le pH, facteur de variation de la densité vermineuse}

Les régressions suivantes ont été retenues, liant le nombre de vers dans leur niche au $\mathbf{p H}$ de celle-ci :

$$
\begin{aligned}
& \mathrm{N}(T, a x e i) \\
& : 438,8 \mathrm{pH}-1124,2 \\
& (\mathrm{p}<0,05) \\
& \mathrm{N} \text { (Habronema spp.) : non significative } \\
& (\mathrm{p}>0,05) \\
& \mathrm{N}(G . \text { intestinalis }) \quad: 7,9 \mathrm{pH}+110,5 \\
& \text { (p }<0,05 \text { ) } \\
& \mathrm{N}(\text { G. nasalis }) \quad:-40,7 \mathrm{pH}+265,2 \\
& (\mathrm{p}=0,02)
\end{aligned}
$$

avec $\mathbf{N}(.)=$. nombre d'individus de l'espèce (...) dans sa localisation préférentielle.

Bien que les données vermineuses ne suivent pas une distribution normale, le nombre élevé d'individus examinés permet d'établir une corrélation sans pratiquer de transformations. Les pH moyens obtenus sur les diverses muqueuses sont relativement élevés (Fundus : 3,57 ; Pylore : 3,96; Proventricule : 4,37) par rapport à ceux obtenus par MALMEJAC (13) chez des animaux fistulisés : 1,5 à 1,3 . Les animaux que nous avons utilisés sont abattus après une diète de 24 à $48 \mathrm{~h}$, ce qui est sans doute la cause de cette discordance.

Le pH mesuré sur les animaux après abattage est en relation avec le nombre de vers présents dans chaque niche sauf pour les habronèmes. Le $\mathrm{pH}$ explique ainsi 7 à $25 \mathrm{p}$. $100 \mathrm{du}$ nombre des vers adultes, selon les espèces. La méthode utilisée par LAGAUTERIE et LEROUX (9) permet d'apprécier la sensibilité des espèces aux variations d'un ou plusieurs facteurs; plus cette sensibilité est faible, plus l'espèce concernée supporte bien les variations du facteur en question. Pour les vers parasites de l'estomac du cheval, les sensibilités aux variations $\mathrm{du} \mathrm{pH}$ sont les suivantes:

$$
\begin{array}{ll}
T \text {. axei } & : 0,308, \\
G . \text { nasalis } & : 0,299,
\end{array}
$$

Habronema spp. : 0,290,

G. intestinalis $: 0,240$.

L'ensemble des espèces est donc peu sensible aux variations du $\mathrm{pH}$. On notera pour $T$, axei, que la sensibilité observée par l'un d'entre nous pour la souche ovine est assez différente $(0,500)$.

L'espèce la moins sensible au $\mathrm{pH}$ est $G$. intestinalis.

\section{CONCLUSION}

Il apparaît que chaque espèce parasitaire a dans l'estomac du cheval sa propre niche écologique bien délimitée, avec des possibilités d'extension sous l'effet de sa propre densité ou de rétraction lors de l'augmentation d'une espèce associée.

Cette étude peut constituer une base pour l'interprétation de lésions parasitaires au niveau de l'estomac du cheval et une référence à une autre nomocénose obtenue pour les mêmes parasites dans une région géographique différente.

Les niches écologiques déterminées que nous venons de définir pourraient être enrichies par l'analyse de paramètres complémentaires tels le taux de matière sèche du contenu stomacal, la pression partielle d'oxygène afin de mieux préciser les dimensions de l'hypervolume.

\section{SUMMARY}

Localisation of parasites in the stomach of horses of the region of Settat (Morocco)

Between October 1977 and September 1978, stomach of ninety-six horses coming from Settat region of Morocco were examined. Each species of parasite was found to have a specific niche in the stomach. Thus Gasterophilus intestinalis larvae were located essentially on cesophagian mucous, $G$, nasalis larvae and Habronema spp. on the pyloric mucous and Trichostrongylus axei particularly on the fundic mucous. In general, the high density of a parasite genus provokes an extension of its ecological niche but on the contrary to it, when the density of other concurrent genera is high there is a retreat towards its usual regular niche.

\section{RESUMEN}

\section{Localización de los parásitos en el estómago del caballo de la región de Settat (Maroc)}

Se realizó el examen de 96 estómagos de caballos proviniendo de la región de Settat (Maroc) de octubre de 1977 a septiembre de 1978. Cada especie parásita tiene un sitio de predilección en el estómago. 
Asi, Gasterophilus intestinalis se localiza esencialmente sobre la mucosa esofágica del estómago y Gasterophilus nasalis sobre la mucosa pilórica; se encuentran Trichostrongylus axei particularmente sobre la mucosa fundica, y Habronema spp. sobre la mucosa pilórica. Por regla general, las densidades elevadas de un género parásito provocan una migración fuera de su sitio ecológico ; al contrario las densidades elevadas de otros géneros competidores imponen una retirada hacia el sitio de predilección habitual.

\section{BIBLIOGRAPHIE}

1. ALPHEY (T.). Studies on the aggregation behaviour of Nippostrongylus brasiliensis. Parasitology, 1971, 63 : 109-117.

2. ALPHEY (T.). Studies on the distributions and site location of Nippostrongylus brasiliensis within the small intestine of laboratory rats. Parasitology, 1979, $61: 449-460$

3. BAWDEN (T. J. W.). Some effects of the diet of mice on Nematospiroides dubius (Nematoda). Parasitology, 1969, 59 : 203-213.

4. CATCOTT (E. J.), SMITHCORPS (J. F.). Equine medicine and surgery. 2nd ed. Illinois, U. S. A., American Veterinary Publication, 1972.

5. CROFTON (H. D.). A quantitative approach to parasitism. Parasitology, 1971, 62 : 178-193.

6. DAJET (J.). Les modèles mathématiques en écologie. Paris, Masson, 1976.

7. DAJET (J.), LECORDIER (C.), LEVÊQUE (L.). Notion de nomocénose : ses applications en écologie. Bull. Soc. Ecol, 1973,3 (4) : 448-462.

8. IWAO (S.), KUNO (E.). Use of regression of mean crowding on mean density for estimating sample size and the transformation of data for the analysis of variance. Res. Pop. Ecol., 1968, $10: 210-214$.

9. LAGAUTERIE (P.), LEROUX (P.). Une méthode d'analyse de la sensibilité aux facteurs ambiants des macro-invertébrés benthiques des eaux courantes. Terre Vie, 1977, 31 (2) : 327-342.
10. LEVIEUX (J.). A propos de la niche écologique. Annls Univ. Abidjan, 1975, Série E, 7 : 7-13.

11. LEWIS (J.), BRYANT (U.). The distribution of Nematospiroides dubius within the small intestine of laboratory mice. J. Helminth., 1976, 50 (3) : 163171.

12. LICHTENFELS $(R$. J.). Helminths of domestic equids. Helminth. Soc. Wash., 1975, 42 (Special issue).

13. MALMEJAC (J.). Eléments de physiologie. 4e éd., Paris, Flammarion, 1970.

14. NEVEU-LEMAIRE (M.). Traité d'entomologie médicale et vétérinaire, Paris, Vigot Frères, 1938.

15. OGBOURNE (C. P.). The prevalence, relative abundance and site distribution of Nematodes of the subfamily Cyathostominae in horses killed in Britain. J. Helminth., 1976, 50 (3) : 203-214.

16. ROSS (J. G.), PURNELL (D. A.), TODD (J. R.). Experimental infection of lambs with Trichostrongylus axei. Res. vet. Sci., 1969, $10: 142-147$.

17. SCHWARTZ (D.). Méthodes statistiques à l'usage des médecins et des biologistes. Paris, Flammarion, 1963.

18. SOMMERVILLE (R. I.). Distribution of some parasitic nematodes in the alimentary tract of sheep, cattle and rabbits. J. Parasit., 1963, 49 : 593-599.

19. SOUTHWOOD (J.). Ecological methods. London, Chapman and Hall, 1971.

20. ZUMPT (T. F.). Myiasis in man and animals in old world. London, Butterworth, 1965. 